\title{
ABSOLUTELY CONTINUOUS COMPONENT OF A CLASS OF INTEGRAL OPERATORS ${ }^{1}$
}

\author{
RICHARD E. SOURS
}

Abstract. The operator $T: L^{2}(0, \infty) \rightarrow L^{2}(0, \infty)$ defined by

$$
T f(x)=\int_{0}^{\infty} \frac{k(x)(k(t))^{-}}{x+t} f(t) d t,
$$

where $(k(t))-$ is the complex conjugate, is studied and conditions are given which are sufficient to characterize the absolutely continuous component.

W. Koppleman [4], J. D. Pincus [5], [6], and M. Rosenblum [9] have studied the selfadjoint, singular-integral operator

$$
S f(x)=a(x) f(x)+(\pi i)^{-1} \mathrm{P} . \mathrm{V} \cdot \int_{E} \frac{b(x)(b(t))^{-}}{x-t} f(t) d t,
$$

where $E$ is a Lebesgue measurable subset of $(-\infty, \infty)$ of positive measure, $a(x)$ is bounded and real valued, and $b(x) \in L^{\infty}(E)$. A complete spectral theory is known for such operators. In particular, the spectral measure is absolutely continuous, except for the possibility of one or two point masses in case $E=(-\infty, \infty)$. Note that formally $S$ has a one-dimensional commutator with the selfadjoint operator $X$ defined by $X f(x)=x f(x)$. For twodimensional commutator systems there is no closed form expression for the spectral invariants (see, however, Pincus [7]). In this paper we study a class of integral operators which formally have a two-dimensional commutator with $X^{2}$, and for which we can calculate the absolutely continuous component.

Define $T: L^{2}(0, \infty) \rightarrow L^{2}(0, \infty)$ by

$$
T f(x)=\int_{0}^{\infty} \frac{k(x)(k(t))^{-}}{x+t} f(t) d t
$$

Received by the editors August 23, 1971.

AMS (MOS) subject classifications (1970). Primary 45E10, 47G05; Secondary 47A55.

Key words and phrases. Integral operator, commutator systems, absolutely continuous component.

1 The content of this paper is part of the author's doctoral dissertation which was written under the direction of Professor James Rovnyak at the University of Virginia, August 1971.

(C) American Mathematical Society 1973 
where $k$ is essentially bounded on $(0, \infty)$. J. S. Howland [2] has given necessary and sufficient conditions that $T$ be of trace class. We now characterize the absolutely continuous component of $T$.

THEOREM. (1) If $x^{-1 / 2-\delta}(1-k(x)) \in L^{2}(0,1)$ and if $x^{-1 / 2+\delta}(1-k(x)) \in$ $L^{2}(1, \infty)$ for some $\delta>0$, then the absolutely continuous component of $T$ has uniform multiplicity 2 on $[0, \pi]$.

(2) If $x^{-1 / 2-\delta}(1-k(x)) \in L^{2}(0,1)$ and if $x^{-1 / 2+\delta} k(x) \in L^{2}(1, \infty)$ for some $\delta>0$, then the absolutely continuous component of $T$ has uniform multiplicity 1 on $[0, \pi]$.

The technique of proof is to find another operator, $T_{1}$, for which the spectral invariants are known and then find conditions on $k$ so that $T_{1}-T$ is of trace class. The Rosenblum-Kato theorem (see Kato [3]) then guarantees that the absolutely continuous components of $T_{1}$ and $T$ are unitarily equivalent. Consider then the operator $T_{1}: L^{2}(0, \infty) \rightarrow L^{2}(0, \infty)$ defined by

$$
T_{1} f(x)=\int_{0}^{\infty}(x+t)^{-1} f(t) d t
$$

Let $L_{v}$ denote the multiplication operator on $L^{2}(-\infty, \infty)$ defined by $L_{v} f(x)=v(x) f(x)$.

LEMMA 1. $T_{1}$ is unitarily equivalent to $L_{v}$, where $v(x)=\pi \operatorname{sech}(\pi x)$. Thus, $T_{1}$ is absolutely continuous and has uniform multiplicity 2 on $[0, \pi]$.

Proof. Let $U: L^{2}(0, \infty) \rightarrow L^{2}(-\infty, \infty)$ be defined by $U f(x)=e^{-x / 2} f\left(e^{-x}\right)$. Then $U$ is unitary, and $U^{-1} f(x)=x^{-1 / 2} f(-\log x)$. Furthermore,

$$
U T_{1} U^{-1} f(x)=e^{-x / 2} \int_{0}^{\infty}\left(e^{-x}+t\right)^{-1} t^{-1 / 2} f(-\log t) d t
$$

By letting $u=-\log t$, we obtain

$$
U T_{1} U^{-1} f(x)=\int_{-\infty}^{\infty} \frac{1}{2} \operatorname{sech}\left(\frac{u-x}{2}\right) f(u) d u .
$$

This convolution is unitarily equivalent, via the Fourier transform, to the desired multiplication operator since the Fourier transform of $\frac{x}{2} \operatorname{sech}(x / 2)$ is $(\pi / 2)^{1 / 2} \operatorname{sech}(\pi x)$ (Erdélyi [1]).

Consider now the operator $T_{2}: L^{2}(0, \infty) \rightarrow L^{2}(0, \infty)$ defined by

$$
T_{2} f(x)=\int_{0}^{\infty} e^{-(x+t) / 2}(x+t)^{-1} f(t) d t .
$$

$T_{2}$ is unitarily equivalent to the Hilbert matrix $[1 /(i+j+1)]_{i, j=0.1,2, \ldots}$, and 
Rosenblum [8] proves that this operator has uniform multiplicity 1 on $[0, \pi]$.

LEMMA 2. Let $\phi(x)$ be essentially bounded on $[0, \infty)$ and define the operator $R: L^{2}(0, \infty) \rightarrow L^{2}(0, \infty)$ by $R f(x)=\int_{0}^{\infty} e^{-x t} \phi(t) f(t) d t$. If $x^{-1 / 2-\delta} \phi(x) \in$ $L^{2}(0,1)$ and $x^{-1 / 2+\delta} \phi(x) \in L^{2}(1, \infty)$, for some $\delta>0$, then $R$ is of trace class.

Proof. The Mellin transform can be used to show that $R$ is a bounded operator.

Let $P_{0}$ be the orthogonal projection mapping $L^{2}(0, \infty)$ onto $L^{2}(0,1)$, and $P_{1}$ be the orthogonal projection mapping $L^{2}(0, \infty)$ onto $L^{2}(1, \infty)$. Then

$$
R f(x)=R P_{0} f(x)+R P_{1} f(x)=\int_{0}^{1} e^{-x t} \phi(t) f(t) d t+\int_{1}^{\infty} e^{-x t} \phi(t) f(t) d t .
$$

Note that $R P_{0}=B_{+} B_{-}$, where $B_{-}: L^{2}(0,1) \rightarrow L^{2}(0,1)$ and $B_{+}: L^{2}(0,1) \rightarrow$ $L^{2}(0, \infty)$ are defined by

$$
\begin{aligned}
& B_{-} f(x)=\int_{x}^{1} t^{-1-\delta} \phi(t) f(t) d t, \quad \text { and } \\
& B_{+} f(x)=\int_{0}^{1} \frac{d}{d t}\left(t^{1+\delta} e^{-x t}\right) f(t) d t .
\end{aligned}
$$

It is easy to see that $B_{+}$and $B_{-}$have square summable kernels, hence they are Hilbert-Schmidt operators and $R P_{0}$ is of trace class. Similarly, $R P_{1}=$ $K_{+}+K_{-}+K_{0}$, where $K_{-}: L^{2}(1, \infty) \rightarrow L^{2}(1, \infty), K_{+}: L^{2}(1, \infty) \rightarrow L^{2}(0, \infty)$ are defined by

$$
\begin{aligned}
& K_{-} f(x)=\int_{x}^{\infty} t^{-1+\delta} \phi(t) f(t) d t, \\
& K_{+} f(x)=\int_{1}^{\infty} \frac{d}{d t}\left(t^{1-\delta} e^{-x t}\right) f(t) d t
\end{aligned}
$$

and $K_{0}$ is the rank one operator defined by

$$
K_{0} f(x)=e^{-x} \int_{1}^{\infty} t^{-1+\delta} \phi(t) f(t) d t .
$$

Again it is easy to see that $K_{+}$and $K_{-}$are Hilbert-Schmidt operators, so $R P_{1}$ is of trace class. Hence $R$ is of trace class.

ProOf of THE THEOREM. To prove part (1) write

$$
T_{1} f(x)=\int_{0}^{\infty}(x+t)^{-1} f(t) d t=\int_{0}^{\infty} e^{-x u} \int_{0}^{\infty} e^{-t u} f(t) d t d u=J_{1}^{2},
$$

where $J_{1}: L^{2}(0, \infty) \rightarrow L^{2}(0, \infty)$ is defined by $J_{1} f(x)=\int_{0}^{\infty} e^{-x t} f(t) d t$. Now 
write $T=J^{*} J$, where $J: L^{2}(0, \infty) \rightarrow L^{2}(0, \infty)$ is defined by $J f(x)=$ $\int_{0}^{\infty} e^{-x t}(k(t))^{-} f(t) d t$. Then $T_{1}-T=J_{1}^{2}-J^{*} J=J_{1}\left(J_{1}-J\right)+\left(J_{1}-J^{*}\right) J$. But $J_{1}-J$ is of trace class by Lemma 2 , so part (1) follows from the RosenblumKato theorem and Lemma 1.

Similarly, for part (2) write $T_{2}=J_{2}^{*} J_{2}$, where $J_{2}: L^{2}(0, \infty) \rightarrow L^{2}(0, \infty)$ is defined by $J_{2} f(x)=\int_{0}^{\infty} e^{-t(1+2 x) / 2} f(t) d t$. Then

$$
T_{2}-T=J_{2}^{*} J_{2}-J^{*} J=J_{2}^{*}\left(J_{2}-J\right)+\left(J_{2}^{*}-J^{*}\right) J .
$$

We use Lemma 2 to show that $J_{2}-J$ is of trace class by setting $\phi(x)=$ $e^{-x / 2}-k(x)$, and noting that the condition $x^{-1 / 2-\delta}(1-k(x)) \in L^{2}(0,1)$ is equivalent to $x^{-1 / 2-\delta}\left(e^{-x / 2}-k(x)\right) \in L^{2}(0,1)$ and $x^{-1 / 2+\delta} k(x) \in L^{2}(1, \infty)$ is equivalent to $x^{-1 / 2+\delta}\left(e^{-x / 2}-k(x)\right) \in L^{2}(1, \infty)$. Part (2) then follows from the Rosenblum-Kato theorem and the result stated above concerning the Hilbert matrix.

\section{REFERENCES}

1. A. Erdélyi et al., Higher transcendental functions. Vol. 1. The hypergeometric function, Legendre functions, McGraw-Hill, New York, 1953. MR 15, 419.

2. J. S. Howland, Trace class Hankel operators, Quart. J. Math. Oxford Ser. (2) 22 (1971), 147-159.

3. T. Kato, Perturbation theory for linear operators, Die Grundlehren der math. Wissenschaften, Band 132, Springer-Verlag, New York, 1966. MR 34 \#3324.

4. W. Koppleman, Spectral multiplicity theory for a class of singular integral operators, Trans. Amer. Math. Soc. 113 (1964), 87-100. MR 29 \#1555a.

5. J. D. Pincus, On the spectral theory of singular integral operators, Trans. Amer. Math. Soc. 113 (1964), 101-128. MR 29 \#1555b.

6. - Commutators, generalized eigenfunction expansions and singular integral operators, Trans. Amer. Math. Soc. 121 (1966), 358-377. MR 32 \#6228.

7. $\longrightarrow$, Commutators and systems of singular integral equations. I, Acta Math. 121 (1968), 219-249. MR 39 \#2026.

8. M. Rosenblum, On the Hilbert matrix. II, Proc. Amer. Math. Soc 9 (1958), 581585. MR 20 \#6038.

9. — A spectral theory for self-ädjoint singular integral operators, Amer. J. Math. 88 (1966), 314-328. MR 33 \#6453.

Department of Mathematics, Wilkes College, Wilkes-Barre, Pennsylvania 18703 